EPJ Web of Conferences 41, 08002 (2013)

DOI: $10.1051 /$ epjconf/20134108002

(C) Owned by the authors, published by EDP Sciences, 2013

\title{
Ultraviolet-resonance femtosecond stimulated Raman study of the initial events in photoreceptor chromophore
}

\author{
S. Takeuchi, H. Kuramochi, and T. Tahara
}

Molecular Spectroscopy Laboratory, RIKEN, 2-1, Hirosawa, Wako 351-0198, Japan

\begin{abstract}
Newly-developed ultraviolet-resonance femtosecond stimulated-Raman spectroscopy was utilized to study the initial structural evolution of photoactive yellow protein chromophore in solution. The obtained spectra changed drastically within $1 \mathrm{ps,}$ demonstrating rapid in-plane deformations of the chromophore.
\end{abstract}

\section{Introduction}

Photoactive yellow protein (PYP), isolated from Halorhodospira halophila, is known to function as a blue-light receptor that is responsible for the negative phototaxis of this organism. The function of this photoreceptor protein is realized by the photocycle that is driven by photoinduced trans-to-cis isomerization of the embedded chromophore (trans-p-coumaric acid, trans-pCA, Figure 1) [1]. So far, numerous experimental efforts have been made to clarify how this subtle structural change in the chromophore ultimately results in the biological response, and the results provided detailed structural insights for transient intermediates appearing during the photocycle, mostly on time scales later than 100 ps. Recent femtosecond infrared experiments have further shown that the first ground-state cis intermediate (the so-called $\mathrm{I}_{0}$ state) is formed in 3 ps [2]. However, we still have very little knowledge on the most important initial isomerization process, i.e., how the chromophore structure evolves after photoabsorption and is converted to the cis form, leaving a missing link between the initially prepared Franck-Condon (FC) state and the experimentally observed $\mathrm{I}_{0}$ state.
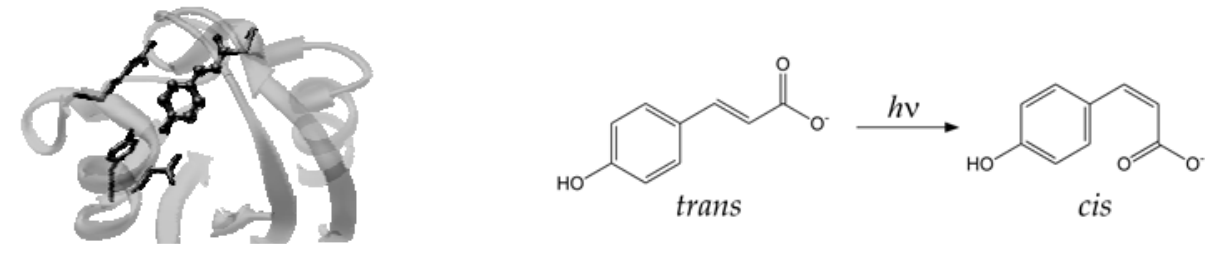

Fig. 1. (Left) Structure of photoactive yellow protein. (Right) Trans-to-cis photoinduced isomerization of a model chromophore, trans-p-coumaric acid (anionic form), in aqueous buffer solution at $\mathrm{pH} 7$.

In exploring the initial events of this photoreception, the primary structural dynamics of the chromophore itself in the fs - ps time range is of extreme interest. Actually, the reported femtosecond absorption and fluorescence measurements for pCA in aqueous buffer solution showed

This is an Open Access article distributed under the terms of the Creative Commons Attribution License 2.0, which permits unrestricted use, distribution, and reproduction in any medium, provided the original work is properly cited. 
that its excited-state dynamics is similar to that in PYP, suggesting that the free trans-pCA is a good model system for the chromophore in PYP. Therefore, it is highly desirable to clarify how transpCA changes its structure in the photoexcited state. Motivated by this idea, we investigated the ultrafast structural dynamics of trans-pCA by using a newly developed ultraviolet resonance femtosecond stimulated Raman spectroscopy (UV-FSRS [3]). Since pCA, both in solution and embedded in the protein pocket, exhibits excited-state absorption exclusively in the uv region, the uv resonance enhancement in femtosecond Raman is essential for selective structural tracking of excited-state pCA.

\section{Experimental}

The FSRS measurements were carried out by using a combination of pump, probe, and Raman pump pulses $[4,5]$. Briefly, the fundamental output of a $1-\mathrm{kHz}$ Ti:sapphire amplifier $(800 \mathrm{~nm})$ was divided into three. The first part was converted to a $300-\mathrm{nm}$ pulse by an OPA and subsequent frequency quadrupling, and the generated uv pulse was used as the pump pulse $(1 \mu \mathrm{J}, 150 \mathrm{fs})$ for photoexcitation of pCA. The second part was focused into a $\mathrm{CaF}_{2}$ plate to generate a white continuum pulse. The near-uv portion $(375-405 \mathrm{~nm})$ was chirp-compensated by a prism pair, and it was used as the probe/reference pulse in the Stokes region. The last part was further divided into two, and they were positively and negatively chirped (with the same magnitude) by two grating dispersive lines. These two counter-chirped pulses were sum-frequency mixed in a BBO crystal to generate a narrow-bandwidth, 400-nm pulse. This ps pulse was used to drive a two-stage BBO OPA tuned to $750 \mathrm{~nm}$, which is seeded by a white continuum pulse after the grating-slit spectral filtering. The OPA output was further frequency-doubled, and the generated narrow-bandwidth uv pulse was used as the Raman pump pulse $\left(375 \mathrm{~nm}, 15 \mathrm{~cm}^{-1}, 1 \mu \mathrm{J}\right)$. The stimulated Raman gain signal, induced by the Raman pump (and the probe), was evaluated from the probe and reference spectra that are measured simultaneously by a spectrograph/CCD system. Trans-p-coumaric acid was dissolved in a phosphate buffer solution at $\mathrm{pH} 7$, where it exists as an anionic form (pCA', Figure 1).

\section{Results and discussion}

Immediately after photoexcitation, $\mathrm{pCA}^{-}$exhibited excited-state absorption at $375 \mathrm{~nm}$ (ESA1, Figure 2). It was found that this transient absorption rapidly blue shifts to $355 \mathrm{~nm}$ (ESA2) within 1 ps. Then, the $355-\mathrm{nm}$ band, as well as a stimulated emission at $430 \mathrm{~nm}$, decayed concomitantly with a time constant of $2.5 \mathrm{ps}$, corresponding to a population decay from the bright $\mathrm{S}_{1}\left(\pi \pi^{*}\right)$ minimum. These transient absorption data indicate that the femtosecond relaxation, which corresponds to the ESA1 - ESA2 spectral shift, occurs in $\mathrm{pCA}^{-}$between the initially prepared FC state and the $\mathrm{S}_{1}\left(\pi \pi^{*}\right)$ minimum.

To examine the femtosecond dynamics, we carried out UV-FSRS measurements for $\mathrm{pCA}^{-}$, where our tunable Raman pump was set to $375 \mathrm{~nm}$ (ESA1 peak) to gain rigorous resonance enhancement exclusively from the excited state (Figure 2). It was found that all the salient changes in the spectral feature occur within the first $1 \mathrm{ps}$, such as (i) a massive frequency upshift for the $812-\mathrm{cm}^{-1}$ and 1573 $\mathrm{cm}^{-1}$ bands, and (ii) a remarkable intensity growth toward $1 \mathrm{ps}$ for the $1150-\mathrm{cm}^{-1}$ and $1475-\mathrm{cm}^{-1}$ bands. These apparent changes in the Raman spectrum observed within 1 ps unequivocally indicate that the structure of excited-state $\mathrm{pCA}^{-}$changes on the femtosecond time scale after photoexcitation. Interestingly, we recognize a striking similarity of the overall spectral pattern between the UV-FSRS and ground-state spectra, suggesting that the molecular structure of excited-state $\mathrm{pCA}^{-}$yet retains the planar-trans conformation, as in the ground state. This is fully consistent with the result of recent ab initio calculations on a PYP model chromophore [6]. In fact, it suggests that the photoexcitation to the $S_{1}$ state is followed by a structural relaxation keeping planar geometry, which ends at the $S_{1}\left(\pi \pi^{*}\right)$ minimum where the $\mathrm{C}_{\mathrm{ph}}-\mathrm{C}_{\mathrm{e}}=\mathrm{C}_{\mathrm{e}}$ angle is smaller than that at the FC state. The present UV-FSRS study strongly suggests that the PYP chromophore in a buffer solution undergoes a rapid in-plane 
deformation, rather than a substantial twist about the $\mathrm{C}_{\mathrm{e}}=\mathrm{C}_{\mathrm{e}}$ bond, and suggests its possible roles, for the chromophore in PYP, in modulating the surrounding hydrogen-bond network in the very initial stage of the photoreception.
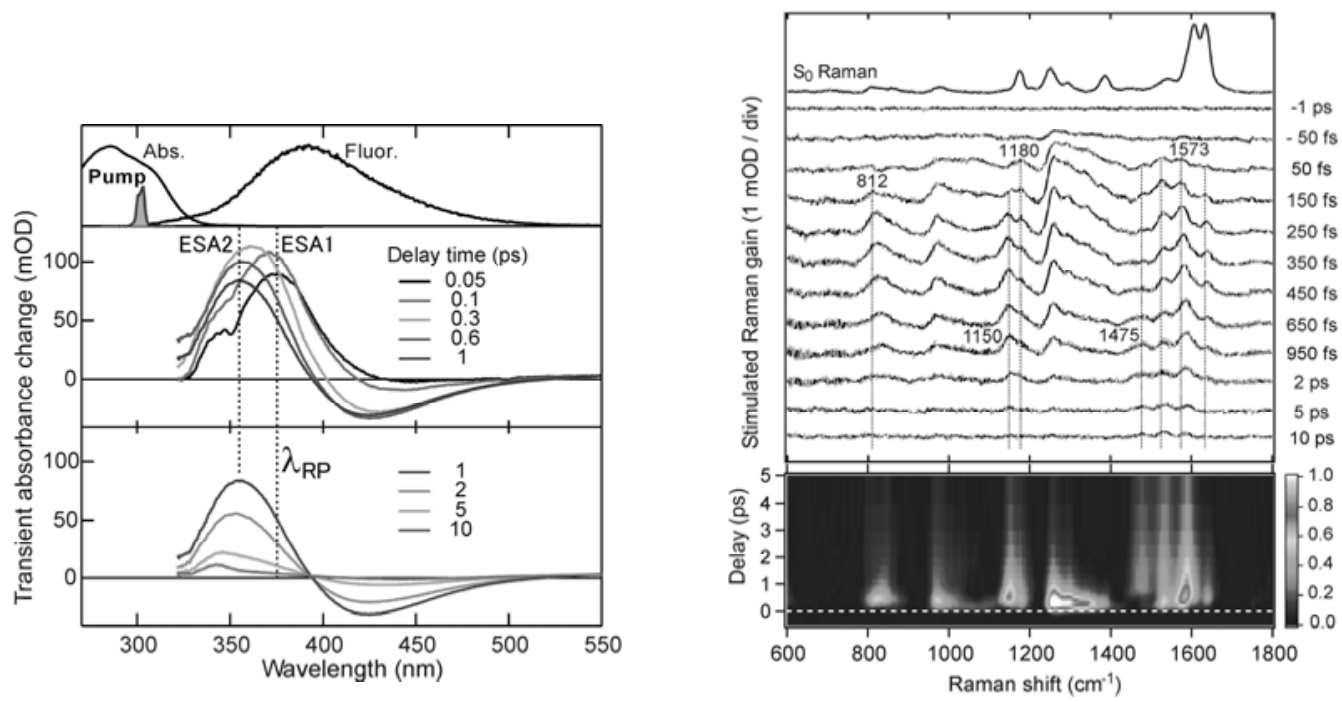

Fig. 2. Femtosecond time-resolved data of $\mathrm{pCA}^{-}$in buffer solution. (Left) Transient absorption spectra (pump: $310 \mathrm{~nm}$ ). (Right) UV-FSRS spectra at several delays together with a ground-state spectrum (pump: $300 \mathrm{~nm}$, Raman pump: $375 \mathrm{~nm}$ ). A 2D contour plot of the UV-FSRS data is also shown.

\section{References}

1. K. J. Hellingwerf, J. Hendriks, T. Gensch, J. Phys. Chem. A 107, 1082 (2003)

2. K. Heyne, O. F. Mohammed, A. Usman, J. Dreyer, E. T. J. Nibbering, M. A. Cusanovich, J. Am. Chem. Soc. 127, 18100 (2005)

3. H. Kuramochi, S. Takeuchi, T. Tahara, J. Phys. Chem. Lett., 3 (15), pp 2025-2029 (2012)

4. M. Yoshizawa, M. Kurosawa, Phys. Rev. A 61, 013808 (1999)

5. P. Kukura, D. W. McCamant, R. A. Mathies, Annu. Rev. Phys. Chem. 58, 461 (2007)

6. E. V. Gromov, I. Burghardt, H. Koppel, L. S. Cederbaum, J. Phys. Chem. A 115, 9237 (2011) 